\title{
Geographic information system methods tell spatiotemporal transmission and its drivers of COVID-19 in Wuhan, China at street-level
}

Wei Liu

Huazhong University of Science and Technology

\section{Wongming Wang}

Huazhong University of Science and Technology

\section{Shuiqiong Hua}

Hubei Provincial Center for Disease Control and Prevention

\section{Cong Xie}

Hubei Provincial Center for Disease Control and Prevention

\section{Bin Wang}

Huazhong University of Science and Technology

\section{Weihong Qiu}

Huazhong University of Science and Technology

\section{Tao Xu}

Huazhong University of Science and Technology

\section{Zi Ye}

Huazhong University of Science and Technology

\section{Linling Yu}

Huazhong University of Science and Technology

\section{Meng Yang}

Huazhong University of Science and Technology

\section{Yang Xiao}

Huazhong University of Science and Technology

\section{Xiaobing Feng}

Huazhong University of Science and Technology

\section{Tingming Shi}

Hubei Provincial Center for Disease Control and Prevention

\section{Mingyan Li}

Hubei Provincial Center for Disease Control and Prevention

\section{Weihong Chen ( $\nabla$ wchen@tjmu.edu.cn )}

Huazhong University of Science and Technology 


\section{Research Article}

Keywords: coronavirus disease 2019; spatiotemporal; SARS-CoV-2; transmission

Posted Date: November 11th, 2020

DOl: https://doi.org/10.21203/rs.3.rs-104986/v1

License: (c) (i) This work is licensed under a Creative Commons Attribution 4.0 International License. Read Full License 


\section{Abstract}

Background: No study has revealed spatial transmission characteristics of COVID-19 in Wuhan, China. We aimed to analyze the spatiotemporal spread of COVID-19 in Wuhan and its influence factors.

Methods: Information of 32,682 COVID-19 cases reported through March 18 were extracted from the national infectious disease surveillance system. Geographic information system methods were applied to analysis transmission of COVID-19 and its influence factors in different periods.

Results: We found decrease in effective reproduction number $(R t)$ and COVID-19 related indicators through taking a series of effective public health measures including restricting traffic, centralized quarantine and strict stay-at home policy. The distribution of COVID-19 cases number in Wuhan showed an obvious global aggregation and a local aggregation in central urban areas, but such aggregations was decreased in the later period of the epidemic. In addition, the analysis at streets-level suggested population density and the number of hospitals were influence factors of spatial difference.

Conclusions: The epidemic situation showed obvious global and local spatial aggregations. High population density and directional flow of the Population to hospitals may account for the aggregations. Strong quarantine measures and restrictions on movement of residents in Wuhan make the epidemic under control in a short time.

\section{Introduction}

Coronavirus disease 2019 (COVID-19), a new type of pneumonia caused by Severe Acute Respiratory Syndrome Coronavirus 2 (SARS-CoV-2), was first reported in early December 2019 in Wuhan, China. In mid-January, some clinicians observed that COVID-19 had strong interpersonal transmission capabilities and could spread through airborne droplets or close contact $[1,2]$. Subsequently, this disease caused an outbreak in Wuhan. The Chinese government included it as a Class B infectious disease on Jan.20, and required to adopt the prevention and control measures for class $A$ infectious disease which is the strictest control measures.

Wuhan has taken some public health intervention measures to control the spread of COVID-19. On Jan.23, the government required all residents to stay at home as much as possible, interrupted public traffic in the city, and suspended all transport links with other areas. During this period, mild cases and close contacts were required to be isolated at home. After Feb.7, all mild COVID-19 cases were required to be centralized treatment in 14 shelter hospitals. At the same time, suspected cases, cases with fever that cannot be ruled out, and close contacts of confirmed cases were isolation in requisitioning hotels. At the same time, suspected cases, cases with fever that cannot be ruled out, and close contacts of confirmed cases were isolation in requisitioning hotels. On Feb.18, all shops were closed and the residents are required to stay at home. Through these measures, the number of new cases fell fast, below 50 on Mar. 8 and no new cases on Mar.18. 
Although previous studies predicted or reconstructed the transmission dynamic of COVID-19 in Wuhan and further discussed the impact of non-pharmaceutical interventions based on COVID-19 epidemic temporal changes [3-6], no study has revealed spatial transmission characteristics. The application of geographic information system (GIS) into routine field epidemiologic surveillance could offer visual evidence for identifying and tracking the spatial spread of infectious diseases [7,8]. In order to learn more from the outbreak in Wuhan, we performed a spatiotemporal analysis of COVID-19 transmission and its driving factors in Wuhan as of Mar. 18, 2020 by using exploratory temporal and GIS methods.

\section{Materials And Methods}

\section{Data source}

COVID-19 was classified as a Class B infectious disease on Jan.20 in China and all infectious cases should be reported immediately through the infectious disease information system according to legal requirements. Information of COVID-19 cases till March 18 were extracted from the national infectious disease surveillance system, which collected birth date, sex, occupation, residential street, date of illness onset (the self-reported date of symptoms such as fever, cough, or other respiratory symptoms), and date of confirmed diagnosis (the laboratory confirmation date of SARS-CoV-2 in the bio-samples or the date on which the clinician determines the case as a clinically diagnosed case). The first-diagnose physician reports case information in a fixed infectious disease report card format. The completeness and accuracy was verified by public health physician in the hospital and then reported to the infectious disease surveillance system. The county-level, city-level and provincial-level CDC completed three reviews and confirmations of the report card within 2 hours, to ensure the accuracy of the data.

The population data (including population size, population density and ratio of the elderly population) were obtained from the statistical yearbooks issued by Wuhan in 2018. The number of public facilities (traffic station, shopping center and hospital) were obtained from Google Maps. Population density is the number of permanent residents per square kilometer; ratio of elderly population was the proportion of the population over 60 years who live permanently in the areas; traffic stations contained both bus stations and subway stations; shopping centers referred to the combinations of retail stores and service facilities in a single building or area that provides comprehensive services to consumers; hospitals with more than 20 beds were included.

The ethics approval was considered exempt because all data collections and analyses belong to a part of continuing public health outbreak investigation determined by the National Health Commission of China.

\section{Case definitions}

Diagnosis of confirmed COVID-19 was conducted according to the diagnostic criteria recommended by the National Health Commission of China [9]. Confirmed case was defined as a patient, with corresponding clinical symptoms and a contact history, who had a positive test of SARS-CoV-2 virus by the real-time reverse-transcription-polymerase-chain-reaction (RT-PCR) assay or high-throughput 
sequencing of nasal and pharyngeal swab specimens. The severity status of the case was categorized as mild, moderate, severe, and critical.

\section{Statistical analysis}

To better reflect the epidemic of COVID-19, the effective reproduction number $(R t)$ was calculated using the method described by a previous publication [10]. The serial interval (mean: 7.5 days, SD: 3.4 days) derived from a reported of first 425 cases in Wuhan [11] were applied to estimate $R t$ and its $95 \%$ coefficient intervals via a 10-days moving average. According to Rt changes in different time, the outbreak was classified into three periods to describe different states of epidemic. Period 1: the time before Jan.24, the pre-cognitive period, when no strong intervention was imposed and the epidemic spread naturally. Period 2: Jan.24-Feb.7, the control period, the spread of COVID-19 was gradually controlled, but the number of cases was still growing (Rt more than 1). Period 3: Feb.8-Mar.18, the transmission fading period, (Rt less than 1 ), when all shops were required to close and the residents were required to stay at home. Cumulative cases, average daily new cases, double time and interval from disease onset to diagnosis in different periods were calculated. The doubling time of COVID-19 in each street was calculated according to the equation introduced by Weon [12]. More specific calculation methods the doubling time and other definitions of COVID-19 indicators were well described in the methods section of the supplementary material.

Since the earliest transmissions may have started at one or several locations, it was not appropriate to use macroscopic spatial statistics to describe the distribution characteristics at this time. So we developed early transmission maps of COVID-19 in Wuhan city by Dec. 25, 2020 to demonstrate the earliest stage of its transmission dynamic. In order to explore the spatial characteristics of COVID-19 spread, we visualized the distribution trend of the onset cases number of each street by constructing a cubic polynomial in different periods on a 3D grid plot. In addition, Moran's I was calculated to reflect the global spatial autocorrelation and local spatial autocorrelation of onset COVID-19 cases number distribution in street-level during different periods. Monte-Carlo method was used to test the significance of Moran's I by simulating 999 times. Cluster map of local indicators of spatial association (LISA) was drawn to show the degree and significance of local cases spatial clustering of one street and its adjacent streets. The modes of local case spatial clustering were divided into five kinds: 1) high-high (area with high cases number surrounded by areas with high cases number), 2) low-low (area with low cases number surrounded by areas with low cases number), 3) low-high (area with low cases number surrounded by areas with high cases number), 4) high-low (area with high cases number surrounded by areas with high cases number), 5) not significant (no significant clustering was found). The calculation method of Moran's I was described in detail in a previous literature [13]. In quest of contribution degree of population density and public facilities in each street to COVID-19 onset cases number, Spatial error model (SEM) was applied to conduct spatial correlation analysis [14].

All analyses were performed with the use of R software (version 3.6.2), ArcGIS 10.2 and GeoDa 1.14.0.0. All two-sided tests were considered as statistically significant when $P$ value was less than 0.05 . 


\section{Results}

\section{Transmission of COVID-19 in 3 time periods}

By March 18, a total of 32,682 cases were identified from the national infectious disease surveillance system (Table S1). Estimates of the effective reproduction number $R t$ through the whole epidemic period was shown in Figure 1. The Rt varied in the period 1 with a peak of 3.86 on Jan. 23, and declined in the period 2 and 3. The $R t$ fell below 1.0 on Feb. 8, 2020 and further decreased to below 0.1 on Mar. 15, 2020. As shown in Table 1, the number of onset cases in three periods were 6,981, 18,381 and 7,320, respectively. Average daily new cases in three periods were 166.2, 1,225.4 and 209.1, respectively. The median of double time elevated from 3.6 days in period 1 to 103.9 days in periods 3 , but the median of interval from disease onset to diagnosis decreased form 20.0 day in period 1 to 3.0 days in period 3 .

\section{The spatiotemporal distribution of COVID-19 cases in Wuhan}

To understand the early transmission of COVID-19 in Wuhan, we made an epidemic map of Wuhan till Dec.25 (Figure 2). We noted that the case first appeared in the center of Wuhan, then extended to the north part after 4 days. On Dec.23, 11 days later, the southern part of Wuhan city also began to report cases, and soon, after 2 days, the east and west parts reported the first case. By Dec.25, 17 days after first reported case, COVID-19 cases had been reported in all 13 districts of Wuhan.

A total of 179 streets in Wuhan city were included in the present analysis and COVID-19 cases were reported from 177 of them. Global spatial trends in whole epidemic and 3 time periods were visualized in Figure 3. The trend lines suggested COVID-19 cases aggregated in central urban area during all periods, but such overall trend of aggregation reduced clearly in period 3. Global spatial autocorrelations in whole epidemic and different periods were examined by Moran's I (Figure 4). In all Moran scatter plots, bubbles mainly aggregated in the first, second and third quadrants, suggested that the spatial distribution form of COVID-19 onset cases in all period were mainly composed of three main patterns: high-high, low-high and low-low. Moran's I in all periods was more than 0 , but dropped from 0.31 in period 1 to 0.12 in period 3 . Significance tests of Moran's I performed by Monte-Carlo method with 999-time simulations indicated significant (pseudo $p$ value $<0.05$ ) global autocorrelation existed in all periods (Figure S1).

In order to have a more intuitive view of spatial distribution of COVID-19 onset cases in different periods, LISA cluster map was employed to graphically demonstrate local autocorrelation of COVID-19 onset cases in street-level (Figure 5). From the perspective of the whole epidemic, the main models of onset cases clustering from the central urban area to the marginal urban area were high-high, high-low or lowhigh, and low-low, successively. As shown in Table 2, the number of streets which did not present significant clustering elevated obviously from 18 in period 1 to 54 in period 3 . Closer inspection of the Table 2 showed such trend of reduction was due to a decrease in both high-high and low-low aggregation patterns.

\section{Analysis of spatial differentiation drivers}


To explore the driving factors of COVID-19 cases spatial differentiation, we performed a tertile analysis of the street according to the population density or the number of public facilities in each street (Table S2). The results suggested that all COVID-19 indictors (including cumulative number of case, average prevalence, doubling time and daily new cases were monotonic increase across tertiles of population density (all $P_{\text {trend }}<0.05$ ). The number of daily new cases in three periods, as well as the average prevalence and the cumulative cases of COVID-19 (all $P_{\text {trend }}<0.05$ ) elevated significantly with the increase in the number of hospitals. We didn't observe any one-way variation trend between shopping center (except number of average daily new cases) and other COVID-19 related indicators, or between the number of traffic station and COVID-19 indicators.

To further validate such potential associations, a spatial error model was constructed to detect the association of the number of COVID-19 onset cases with population density, ratio of the elderly population and number of public facilities in street-level. As shown in Table 3, population density and the number of hospitals were significantly associated with the number of onset cases at street-level (both $P$ $<0.05$ ) rather than ratio of elderly population and the number of other public facilities throughout the whole epidemic. When stratified into three periods, significant associations of onset cases with population density and the number of hospitals were observed in period 1 and 2 . In addition, the number of traffic stations was positively associated with onset cases with a coefficient of 4.437 in period 2 . Strikingly, no significant association between population density and onset cases was found in period 3 . Nonetheless, the number of hospitals was still positive associated with onset cases elevation in period 3 , but the coefficient was lower than that in period 2 (6.809 vs 13.559).

\section{Discussion}

The present study found that the transmission of COVID-19 in Wuhan experienced three periods of outbreak, control and decline in time, and presented a spatial clustering in the central urban distrain. In addition, population density and the number of hospitals were both positive associated with COVID-19 indicators at streets-level.

In the early stage, it took 17 days from the first case to cases appeared in all districts in Wuhan. The $R t$ reached a peak on Jan.23, 46 days from the first case reported. The government intervened with a series of public health measures after the discovery of conclusive evidence that COVID-19 could be passed from person to person [15]. The present study divided the epidemic of COVID-19 in Wuhan into three periods. In period 1, when no strong intervention was implemented, the doubling time of COVID-19 cases was 3.6 days, which was shorter than the 7.5 (5.3-19) days calculated by model simulations in an earlier study [11]. Such difference may be due to the limitation of detection capacity in the early stage of the outbreak, resulting some cases not being confirmed in a timely manner and the transmission not being properly assessed. In period 2, indicators of transmission, including onset cases and average daily new cases indicated that the epidemic was still rising, but changes in doubling time and $R t$ both suggested the epidemic was under control in some degree. On one hand, as the median incubation period of COVID-19 is up to 14 days [16,17], so changes in indicators may lag behind the impact of intervention measures. On 
the other hand, mild and suspected cases were required to isolate at home during that period, which still had a great risk of transmission, especially in areas with high population density. In period 3 , the doubling time increased more than 10 times that of the previous period. In fact, almost all of the identified potential infectors were isolated during the period 3 , and the strict stay-at-home policy for all residences cut off transmission to a great extent. Therefore, more stringent measures to isolate and limit population movements, rather than just restricting public transportation and population gathering, are needed to control the outbreak of COVID-19 in a short time.

The present study found that the epidemic situation showed obvious aggregation in central urban areas, where found the first case. In three periods, significant spatial autocorrelations of COVID-19 onset cases number in Wuhan were found, especially in period 1 and 2 . The transmission of COVID-19 in first two periods tended to spread from high-incidence areas to low-incidence areas. The size of aggregation reduced in the later stage (after the implementation of strict population movement control measures, period 3) of the epidemic. Such a change in spatial distribution characteristics suggested that the movement of people during an epidemic should be limited to the greatest extent, especially in highincidence areas.

Our study also found that the population density as well as the number of hospitals in the streets may be driver factors of spatial differences. In addition, our research confirmed that high population density and higher number of hospitals facilitated the spread of COVID-19 in street, and streets with low population density and higher hospitals number had lower daily new cases and attack rate. Due to the emergence of huge cases, the hospital may become a source of infection. In fact, a large number of panicked residences with similar or suspected symptoms of COVID-19 flocked to hospitals to seek for treatment, which not only led to the directional movement of cases, but also increased the risk of cross-infections. An analysis of 138 COVID-19 cases conducted by a hospital in Wuhan showed that the ratio of hospital infection was up to $41.3 \%$ [18], which also suggested a high risk of hospital infection. In addition, a number of public health interventions were implemented by the Wuhan government from Jan.23 to Feb.18, including shutdown of public gathering places, restrictions of inner-city traffic, and strict stay-athome policy for all residences. These effective interventions might lead to the fact we did not observe the association of traffic stations with increased number of average daily new cases. Restricting traffic eliminated the impact of the number of stations on COVID-19. Furtherly, no relationship was found between prevalence and the number of shopping center. These results suggested that interventions have a strong ability to control the epidemic of COVID-19. It is surprising that no association was observed between ratio of elderly population and the number of onset cases, even though multiple studies [3-6] and our results jointly confirmed the susceptibility of elderly to COVID-19. We thought that it may be because area with ratio of elderly population had lower population density and some of them are located in remote areas [19]. The lower population density and population mobility resulted in the reduced a lower probability of infection among the residents in these areas.

The present study performed a spatiotemporal analysis of the COVID-19 transmission in Wuhan, China for the first time. There are some limitations in this study. As the data source is the national infectious 
disease surveillance system, the statistics of the number of cases in each street are based on the current address of the reported cases rather than the contact history, which may lead to a decrease in the authenticity of the number of cases in each street. However, given a series of measures to limit population movement was taken relatively early in the outbreak, our study was deemed to be of good credibility.

\section{Conclusion}

In the early stage of the epidemic, COVID-19 spread rapidly in Wuhan and showed a trend of spreading from downtown to surrounding areas. The epidemic situation shows obvious aggregation in central city. High population density and high number of hospitals may be risk factors for the transmission of the COVID-19 in Wuhan. The spatiotemporal analysis of COVID-19 transmission in Wuhan proves that reducing the movement of population as far as possible and stronger isolation have a significant effect on containing the transmission.

\section{Declarations}

Acknowledgments: We are grateful to all staff at the national, provincial, and municipal Center for Disease Control and Prevention for providing the data and all medical staff members and field workers who are working on the frontline of caring for patients and collecting the data. All members of study team are greatly acknowledged.

Funding: This work was supported by the Major Research Program of the National Natural Science Foundation of China (91843302) and Wuhan Municipal Health Committee Emergency Fund for COVID-19 (EG20B04). The funders did not play any role in study design; in the collection, analysis, and interpretation of data; in the writing of the report; nor in the preparation, review, or approval of the manuscript.

Authors' contributions: Wei Liu and Dongming Wang contributed to data analysis, interpreted the results and wrote the manuscript. Shuiqiong Hua, Cong Xie and Mingyan Li helped to collect data and revised the manuscript. Bin Wang, Weihong Qiu, Tao Xu, Zi Ye, Linling Yu, Meng Yang, Yang Xiao, Xiaobing Feng and Tingming Shi helped to data analysis and revised the manuscript. Weihong Chen designed the study, interpreted the results, and revised the manuscript.

Ethics approval: Not applicable.

Declarations of interest: The authors declare no conflict of interest. 


\section{References}

1. Wu P, Hao X, Lau EHY, et al. Real-time tentative assessment of the epidemiological characteristics of novel coronavirus infections in Wuhan, China, as at 22 January 2020. Euro surveillance : bulletin Europeen sur les maladies transmissibles = European communicable disease bulletin. 2020;25(3):2000044. doi:10.2807/1560-7917.ES.2020.25.3.2000044

2. Carlos WG, Dela Cruz CS, Cao B, Pasnick S, Jamil S. Novel Wuhan (2019-nCoV) Coronavirus. Am J Respir Crit Care Med. 2020;201(4):P7-p8. doi:10.1164/rccm.2014P7

3. Kucharski AJ, Russell TW, Diamond C, et al. Early dynamics of transmission and control of COVID19: a mathematical modelling study. The Lancet. Infectious diseases. 2020. doi:10.1016/s14733099(20)30144-4

4. Wu JT, Leung K, Leung GM. Nowcasting and forecasting the potential domestic and international spread of the 2019-nCoV outbreak originating in Wuhan, China: a modelling study. Lancet. 2020;395(10225):689-97. doi:10.1016/s0140-6736(20)30260-9

5. Pan A, Liu L, Wang C, Guo H, et al. Association of Public Health Interventions With the Epidemiology of the COVID-19 Outbreak in Wuhan, China. JAMA. 2020 May 19;323(19):1915-1923. doi: 10.1001/jama.2020.6130

6. Hao X, Cheng S, Wu D, Wu T, Lin X, Wang C. Reconstruction of the full transmission dynamics of COVID-19 in Wuhan. Nature. 2020 Aug;584(7821):420-424. doi: 10.1038/s41586-020-2554-8

7. Lai PC, Wong CM, Hedley AJ, et al. Understanding the spatial clustering of severe acute respiratory syndrome (SARS) in Hong Kong. Environ Health Perspect. 2004 Nov;112(15):1550-6. doi: 10.1289/ehp.7117

8. Higgs G, Gould M. Is there a role for GIS in the 'new NHS'? Health Place. 2001 Sep;7(3):247-59. doi: 10.1016/s1353-8292(01)00014-4

9. National Health Commission of the Peoples's Republic of China. Clinical diagnosis and treatment guidance of 2019 novel coronavirus (COVID-19) caused pneumonia. 2020.

http://www.nhc.gov.cn/yzygj/s7653p/202002/3b09b894ac9b4204a79db5b8912d4440. Accessed 15 Mar 2020.

10. Cori A, Ferguson NM, Fraser C, Cauchemez S. A new framework and software to estimate timevarying reproduction numbers during epidemics. Am J Epidemiol. 2013 Nov 1;178(9):1505-12. doi: 10.1093/aje/kwt133

11. Li Q, Guan X, Wu P, et al. Early Transmission Dynamics in Wuhan, China, of Novel CoronavirusInfected Pneumonia. The New England journal of medicine. 2020;382(13):1199-207. doi:10.1056/NEJMoa2001316

12. Weon BM. Doubling time tells how effective Covid-19 prevention works. medRxiv. 2020:2020.03.26.20044644. doi:10.1101/2020.03.26.20044644

13. Yu H, Gong H, Chen B, Liu K, Gao M. Analysis of the influence of groundwater on land subsidence in Beijing based on the geographical weighted regression (GWR) model. Sci Total Environ. 2020 Oct 
10;738:139405. doi:10.1016/j.scitotenv.2020.139405

14. Li W, Gu Y, Liu F, Li C. The effect of command-and-control regulation on environmental technological innovation in China: a spatial econometric approach. Environ Sci Pollut Res Int. 2019 Dec;26(34):34789-34800. doi: 10.1007/s11356-018-3678-3

15. Chan JF-W, Yuan S, Kok K-H, et al. A familial cluster of pneumonia associated with the 2019 novel coronavirus indicating person-to-person transmission: a study of a family cluster. The Lancet. 2020;395(10223):514-23. doi:https://doi.org/10.1016/S0140-6736(20)30154-9

16. Guan W-J, Ni Z-Y, Hu Y, et al. Clinical Characteristics of Coronavirus Disease 2019 in China. The New England journal of medicine. 2020. doi:10.1056/NEJMoa2002032

17. Lauer SA, Grantz KH, Bi Q, et al. The Incubation Period of Coronavirus Disease 2019 (COVID-19) From Publicly Reported Confirmed Cases: Estimation and Application. Ann Intern Med. 2020. doi:10.7326/m20-0504

18. Wang D, Hu B, Hu C, et al. Clinical Characteristics of 138 Hospitalized Patients With 2019 Novel Coronavirus-Infected Pneumonia in Wuhan, China. Jama. 2020. doi:10.1001/jama.2020.1585

19. Wuhan Civil Affairs Bureau. Wuhan population Aging Report 2016. http://mzj.wuhan.gov.cn/mzdt_912/bsmz_913/201701/t20170126_156925.shtml Accessed 15 Mar 2020.

\section{Tables}

Table 1. Transmission of COVID-19 in Wuhan during different period

\begin{tabular}{|llll|}
\hline Variables & $\begin{array}{l}\text { Before Jan.24, } \\
\mathbf{2 0 2 0}\end{array}$ & $\begin{array}{l}\text { Jan.24- Feb.7, } \\
\mathbf{2 0 2 0}\end{array}$ & $\begin{array}{l}\text { After Feb.7, } \\
\mathbf{2 0 2 0}\end{array}$ \\
\hline Onset cases, $\mathrm{n}$ & 6,981 & 18,381 & 7,320 \\
\hline Average daily new cases, $\mathrm{n}$ & 166.2 & 1225.4 & 209.1 \\
\hline Double time, day & 3.6 & 8.1 & 103.9 \\
\hline $\begin{array}{l}\text { Interval from disease onset to diagnosis, } \\
\text { median (IQR), day }\end{array}$ & $\begin{array}{l}20.0(14.0- \\
25.0)\end{array}$ & $11.0(8.0-16.0)$ & $3.0(1.0-5.0)$ \\
\hline
\end{tabular}

Abbreviations: COVID-19, coronavirus disease 2019; IQR, interquartile range.

Table 2. Street-level spatial clustering models of COVID-19 onset cases in different periods 


\begin{tabular}{|llllll|}
\hline Period & \multicolumn{5}{l|}{ Number of different spatial clustering models } \\
\cline { 2 - 6 } & High-high & High-low & Low-high & Low-low & Not significant \\
\hline The whole epidemic & 68 & 4 & 43 & 37 & 27 \\
\hline Period 1 & 69 & 4 & 41 & 47 & 18 \\
\hline Period 2 & 70 & 5 & 43 & 39 & 22 \\
\hline Period 3 & 59 & 3 & 39 & 24 & 54 \\
\hline
\end{tabular}

Significance of local spatial clustering was tested by local Moran's I. Period 1, the pre-cognitive period, when COVID-19 spread without strong inventions. Period 2, the control period, the spread of COVID-19 is gradually being controlled, but the number of cases is still growing (Rt more than 1 ). Period 3 , the transmission fading period (Rt less than 1).

Table 3. Street-level correlation of COVID-19 cases number with population density and the number of public facilities of Wuhan city in different periods 


\begin{tabular}{|c|c|c|c|c|c|}
\hline Periods & Characteristics & Coefficient & $\begin{array}{l}\text { Std. } \\
\text { Error }\end{array}$ & $\begin{array}{l}z- \\
\text { value }\end{array}$ & $\begin{array}{l}P \text { value for } \\
\text { Coefficient }\end{array}$ \\
\hline \multirow{5}{*}{$\begin{array}{l}\text { The whole } \\
\text { epidemic }\end{array}$} & Population density & 0.003 & 0.001 & 4.961 & $<0.01$ \\
\hline & $\begin{array}{l}\text { Ratio of the elderly } \\
\text { populations }\end{array}$ & -0.468 & 3.453 & -0.136 & 0.892 \\
\hline & Number of traffic stations & 6.439 & 3.617 & 1.780 & 0.075 \\
\hline & $\begin{array}{l}\text { Number of shopping } \\
\text { centers }\end{array}$ & -5.152 & 4.824 & -1.068 & 0.286 \\
\hline & Number of hospitals & 25.373 & 7.042 & 3.603 & $<0.01$ \\
\hline \multirow[t]{5}{*}{ Period 1} & Population density & 0.001 & 0.001 & 4.852 & $<0.01$ \\
\hline & $\begin{array}{l}\text { Ratio of the elderly } \\
\text { populations }\end{array}$ & -0.335 & 0.793 & -0.059 & 0.953 \\
\hline & Number of traffic stations & 1.616 & 0.827 & 1.954 & 0.051 \\
\hline & $\begin{array}{l}\text { Number of shopping } \\
\text { centers }\end{array}$ & -1.403 & 1.103 & -1.273 & 0.203 \\
\hline & Number of hospitals & 5.119 & 1.609 & 3.182 & $<0.01$ \\
\hline \multirow[t]{5}{*}{ Period 2} & Population density & 0.002 & 0.001 & 5.157 & $<0.01$ \\
\hline & $\begin{array}{l}\text { Ratio of the elderly } \\
\text { populations }\end{array}$ & -1.336 & 1.943 & -0.687 & 0.492 \\
\hline & Number of traffic stations & 4.437 & 2.033 & 2.183 & $<0.05$ \\
\hline & $\begin{array}{l}\text { Number of shopping } \\
\text { centers }\end{array}$ & -3.324 & 2.711 & -1.226 & 0.220 \\
\hline & Number of hospitals & 13.559 & 3.957 & 3.427 & $<0.01$ \\
\hline \multirow[t]{5}{*}{ Period 3} & Population density & 0.001 & 0.001 & -0.358 & 0.720 \\
\hline & $\begin{array}{l}\text { Ratio of the elderly } \\
\text { populations }\end{array}$ & 1.456 & 1.017 & 1.432 & 0.152 \\
\hline & Number of traffic stations & 0.428 & 1.117 & 0.384 & 0.701 \\
\hline & $\begin{array}{l}\text { Number of shopping } \\
\text { centers }\end{array}$ & -0.545 & 1.497 & -0.364 & 0.716 \\
\hline & Number of hospitals & 6.809 & 2.191 & 3.107 & $<0.01$ \\
\hline
\end{tabular}

Spatial error model was applied to detect the correlation of COVID-19 cases number with population density and the number of public facilities. Period 1, the pre-cognitive period, when COVID-19 spread without strong inventions. Period 2, the control period, the spread of COVID-19 is gradually being 
controlled, but the number of cases is still growing ( $R t$ more than 1$)$. Period 3 , the transmission fading period (Rt less than 1).

\section{Figures}

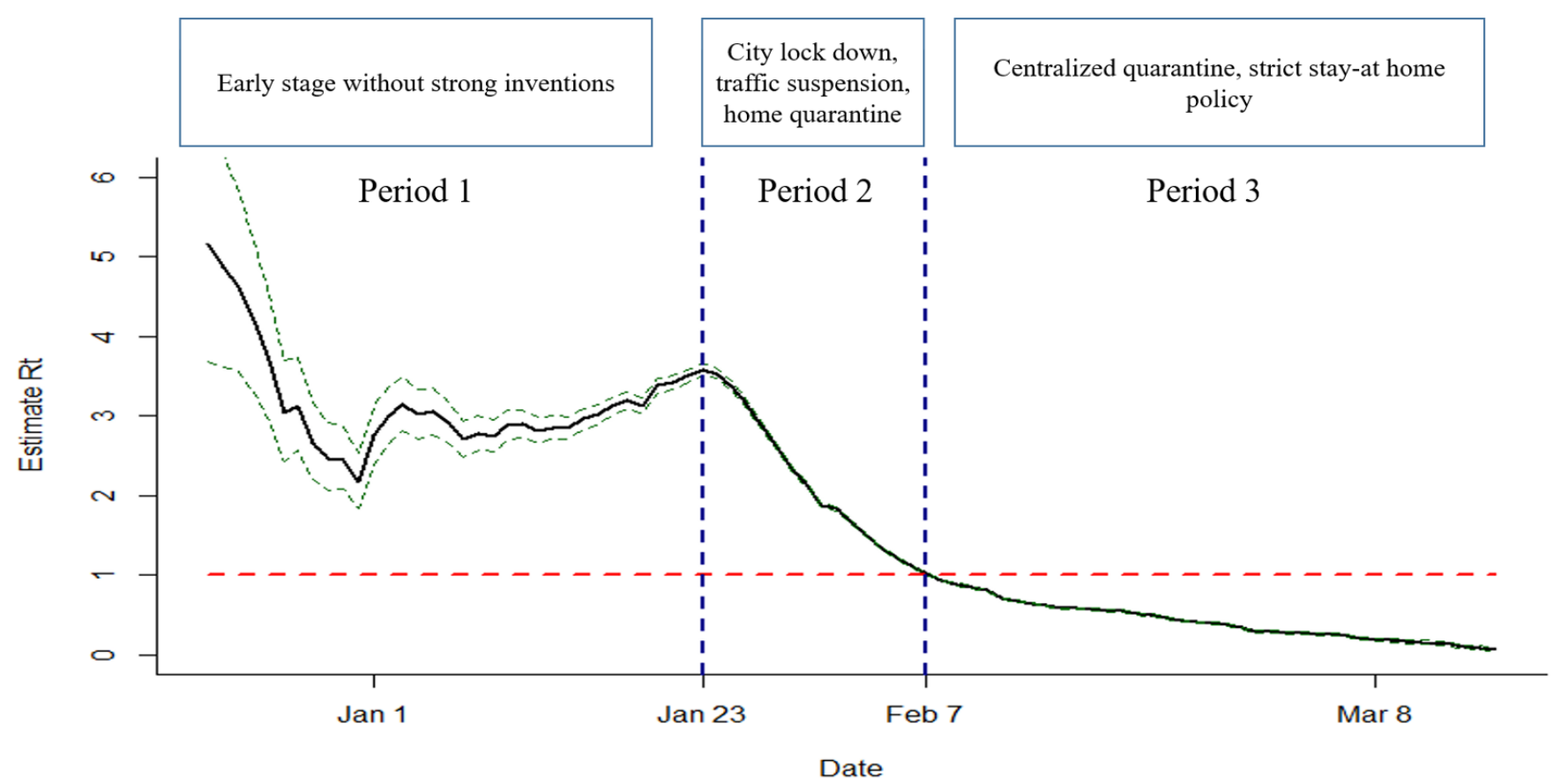

\section{Figure 1}

The Effective Reproduction Number (Rt) Estimates Based on Coronavirus Disease 2019 (COVID-19) Cases in Wuhan, China. Period 1: the pre-cognitive period, when COVID-19 spread without strong inventions. Period 2: the control period, the spread of COVID-19 is gradually being controlled, but the number of cases is still growing (Rt more than 1). Period 3: the transmission fading period (Rt less than 1). 


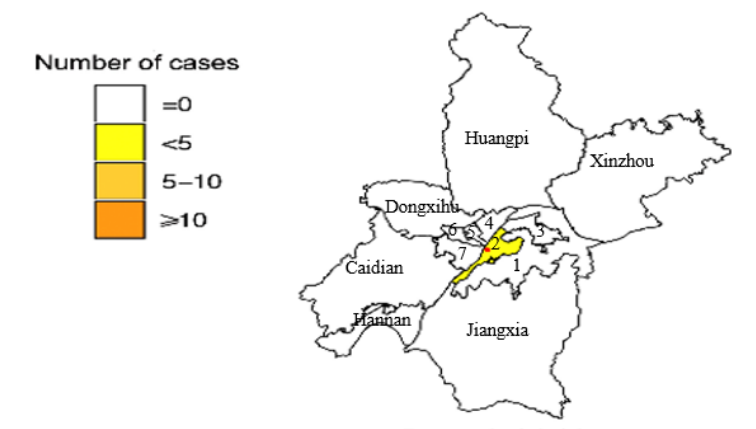

Dec. 8,2019

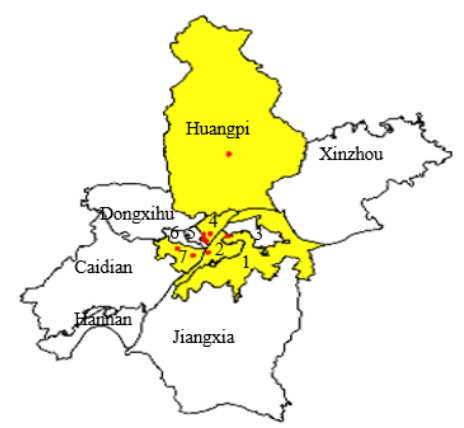

Dec. 15,2019

Central urban districts

1 Hongshan

2 Wuchang

3 Qingshan

4 Jiang'an

5 Jianghan

6 Qiaokou

7 Hanyang

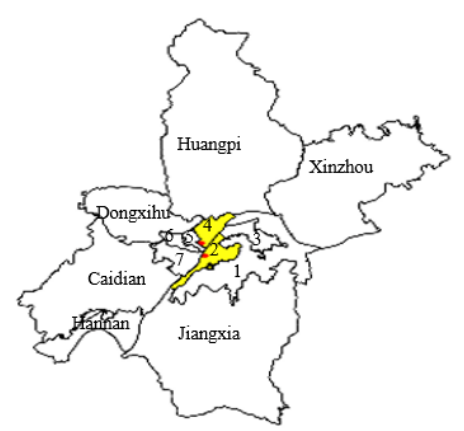

Dec. 11,2019

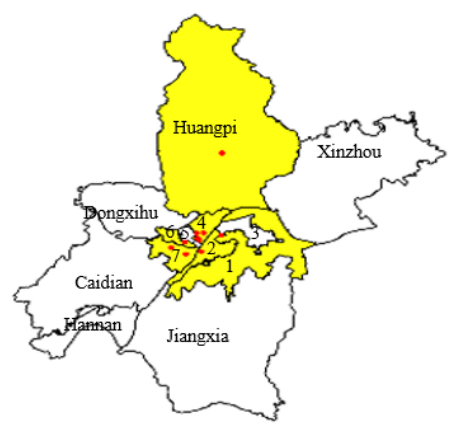

Dec. 16,2019

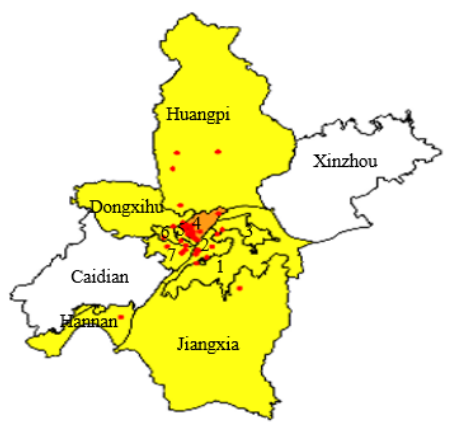

Dec. 23,2019

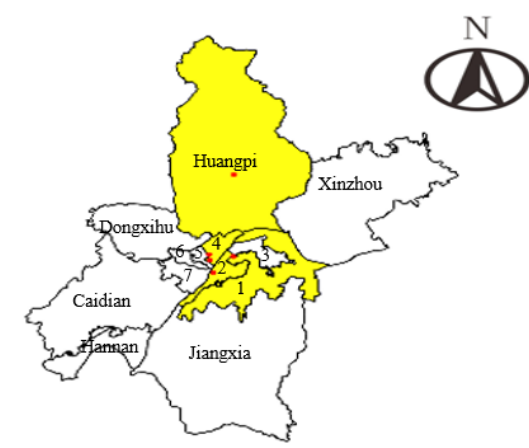

Dec. 12,2019

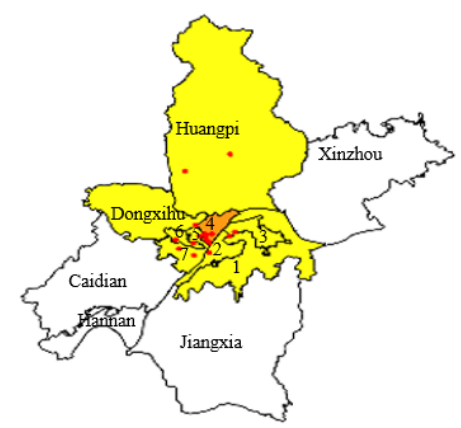

Dec. 17,2019

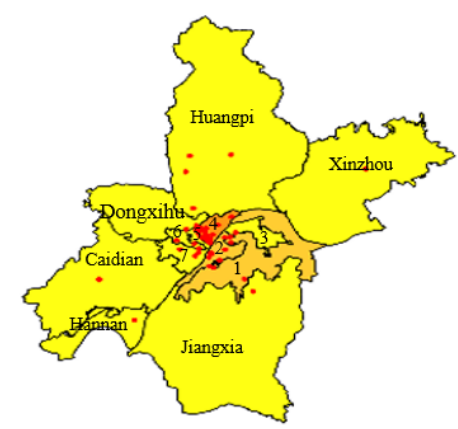

Dec. 25,2019

Figure 2

Early Transmission of COVID-19 in Wuhan, China. The red dots represent the first cases in each street. Note: The designations employed and the presentation of the material on this map do not imply the expression of any opinion whatsoever on the part of Research Square concerning the legal status of any country, territory, city or area or of its authorities, or concerning the delimitation of its frontiers or boundaries. This map has been provided by the authors. 

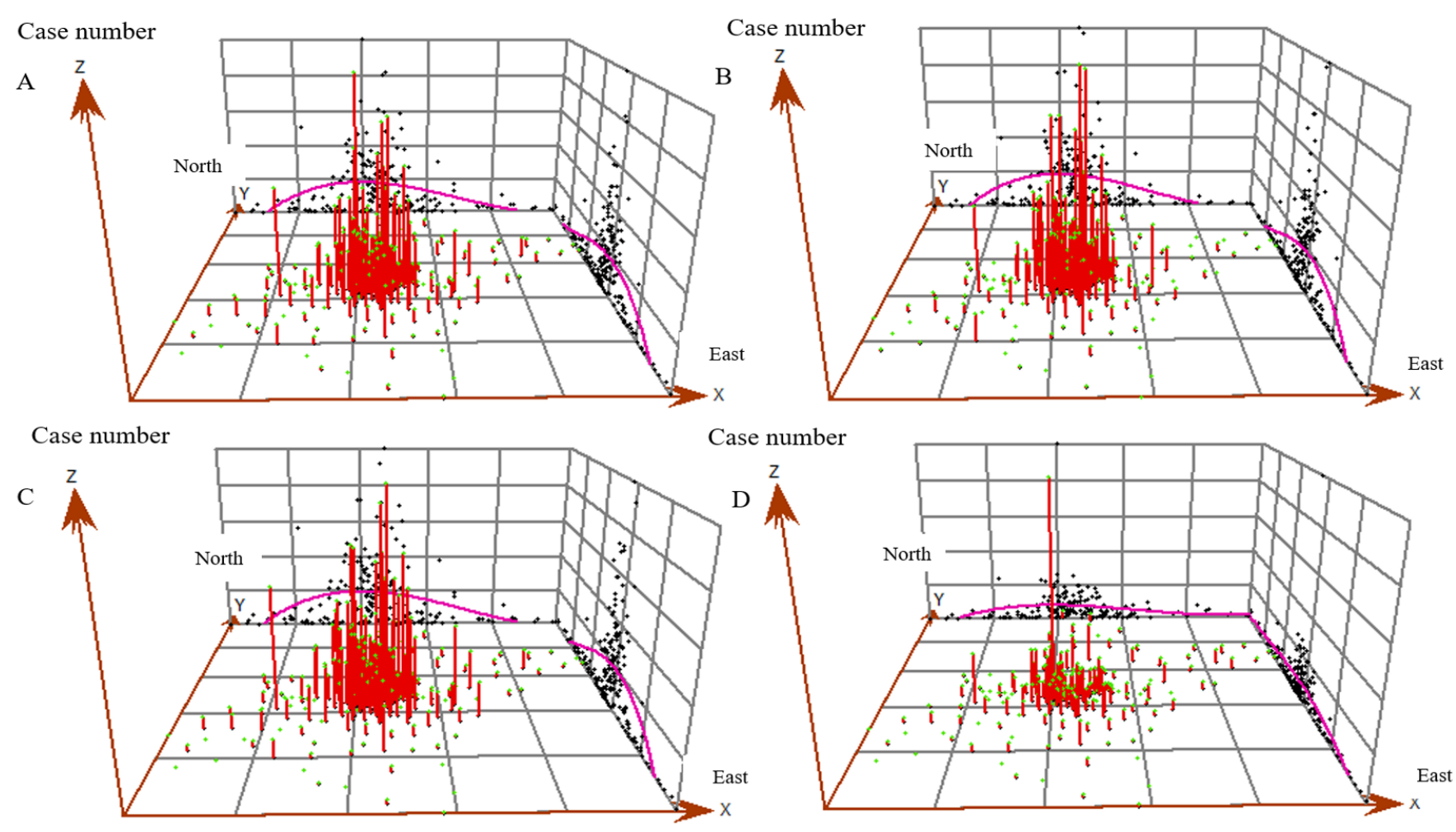

\section{Figure 3}

Street-level global spatial trend of onset COVID-19 cases Wuhan, China in different periods, respectively. A: The whole epidemic time (from Dec. 8, 2019 to Mar. 18, 2020). B: Period 1, the pre-cognitive period, when COVID-19 spread without strong inventions. C: Period 2, the control period, the spread of COVID-19 is gradually being controlled, but the number of cases is still growing (Rt more than 1). D: Period 3, the transmission fading period (Rt less than 1). The X-axis points to the north of Wuhan, the Y-axis points to the east of Wuhan, and the Z-axis is cases number. The points on the grid are projections of cases number in each street. The curve on the grid shows the distribution trend of cases in overall city. The red column represents the cases number in each street. 

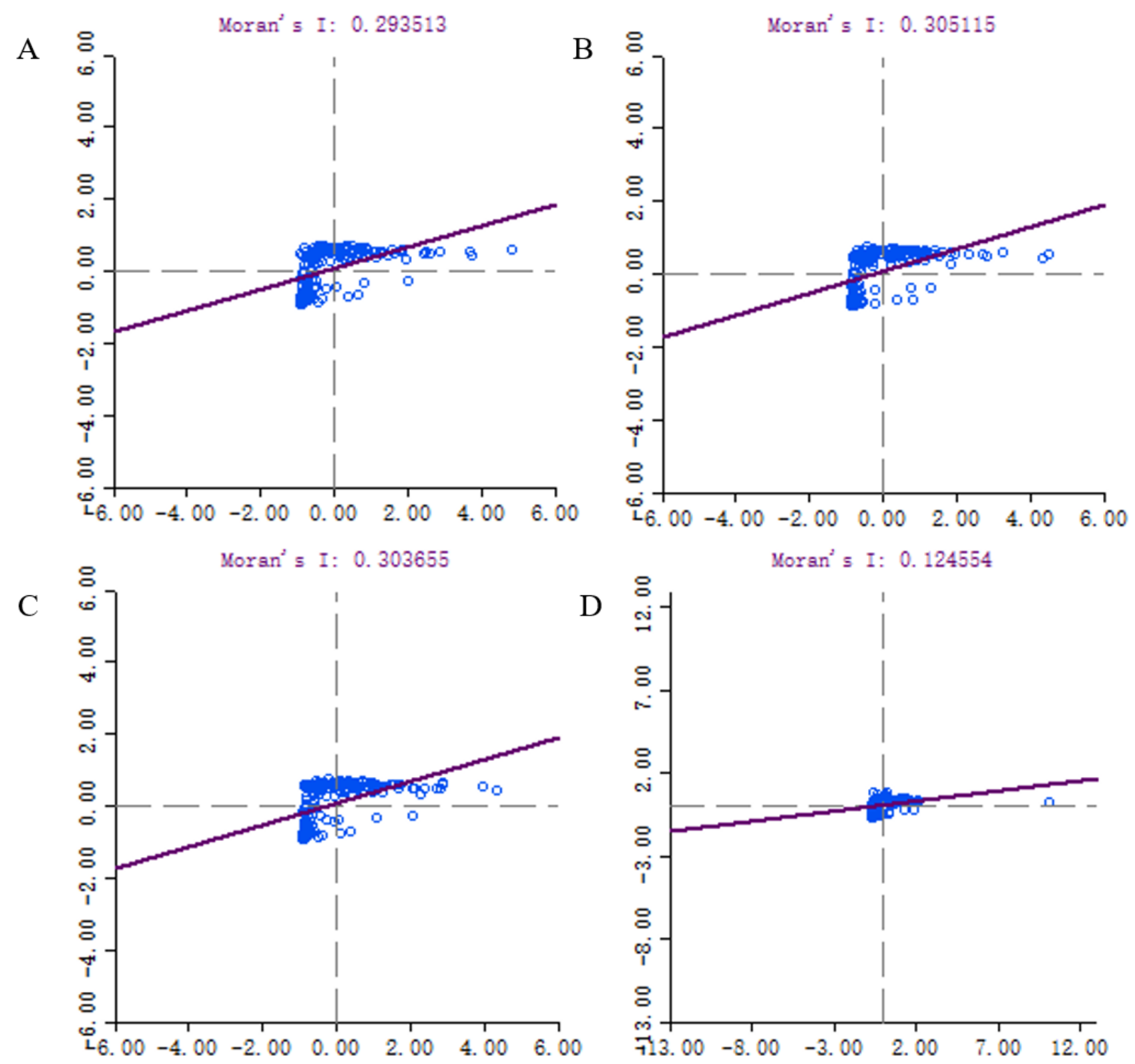

\section{Figure 4}

Moran scatter plot of onset COVID-19 cases spatial autocorrelation in streets of Wuhan city. The X-axis is the standardized value of cases number, and the $Y$-axis is the standardized value of the cases number in adjacent streets. The bubbles represent all streets of Wuhan city. A: The whole epidemic time (from Dec. 8, 2019 to Mar. 18, 2020). B: Period 1, the pre-cognitive period, when COVID-19 spread without strong inventions. C: Period 2, the control period, the spread of COVID-19 is gradually being controlled, but the number of cases is still growing (Rt more than 1). D: Period 3, the transmission fading period (Rt less than 1). 


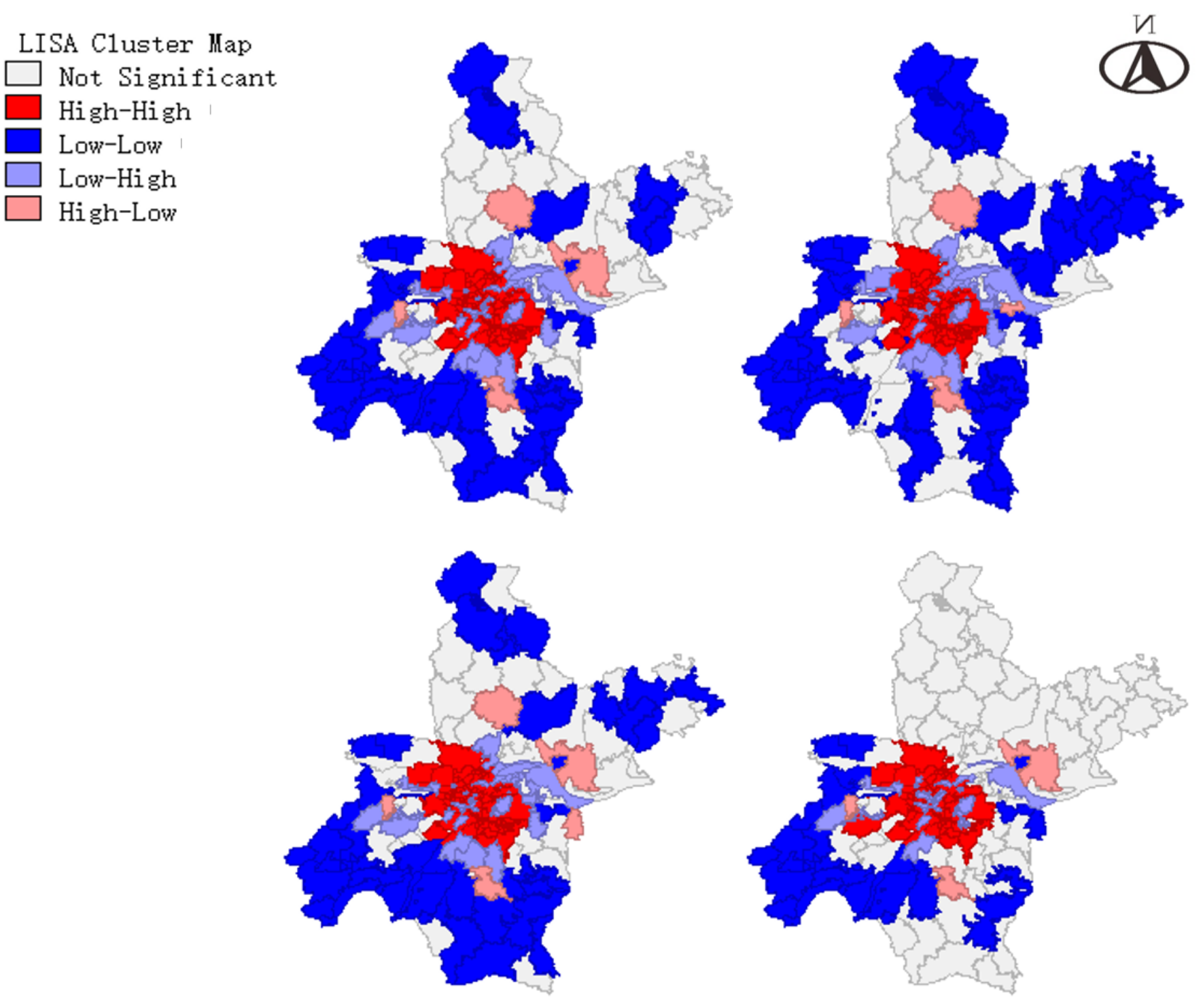

\section{Figure 5}

Lisa cluster map of onset COVID-19 cases local spatial autocorrelation of Wuhan city in street-level. A: The whole epidemic time (from Dec. 8, 2019 to Mar. 18, 2020). B: Period 1, the pre-cognitive period, when COVID-19 spread without strong inventions. C: Period 2, the control period, the spread of COVID-19 is gradually being controlled, but the number of cases is still growing (Rt more than 1 ). D: Period 3 , the transmission fading period (Rt less than 1). Note: The designations employed and the presentation of the material on this map do not imply the expression of any opinion whatsoever on the part of Research Square concerning the legal status of any country, territory, city or area or of its authorities, or concerning the delimitation of its frontiers or boundaries. This map has been provided by the authors.

\section{Supplementary Files}


This is a list of supplementary files associated with this preprint. Click to download.

- supplementarymaterial.docx 